\title{
REGISTRO DE Pseudis paradoxa (Linnaeus, 1758) (ANURA, PSEUDIDAE) EN EL DEPARTAMENTO DE SUCRE, COLOMBIA
}

\section{RECORD OF Pseudis paradoxa (Linnaeus, 1758) (ANURA, PSEUDIDAE), IN THE DEPARTMENT OF SUCRE, COLOMBIA}

\author{
DE LA OSSA V. JAIME ${ }^{1 *}$, SAMPEDRO-MARIN, ALCIDES ${ }^{1}$, GALVÁN- \\ GUEVARA, SILVIA ${ }^{2}$, y DE LA OSSA-LACAYO, ALEJANDRO ${ }^{3}$ \\ ${ }^{1}$ Profesor Universidad de Sucre, Colombia, Grupo de Investigación en \\ Biodiversidad Tropical. ${ }^{2}$ Grupo de Investigación en Biodiversidad Tropical, \\ Universidad de Sucre, Colombia. ${ }^{3}$ Universidad Javeriana de Colombia \\ *Correspondencia: jaimedelaossa@yahoo.com
}

El 20 de octubre y el 3 de noviembre de 2008 fueron recolectados sendos ejemplares de Pseudis paradoxa, los que fueron entregados a investigadores del Grupo de Biodiversidad Tropical, de la Universidad de Sucre. El hecho causó expectación en la población sucreña porque se trataba de dos individuos metamórficos, que en esta especie presentan la cola muy grande, lo que les da un aspecto extraño, que fue interpretado como algo sobrenatural por los recolectores y personas de esas zonas. Un espécimen (20 de octubre) fue colectado en el jagüey de la granja El Perico, de la Universidad de Sucre,

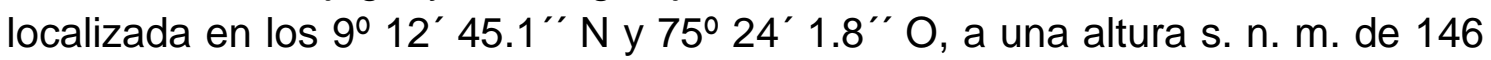
$\mathrm{m}$ y perteneciente políticamente al municipio de Sampués, departamento de Sucre, Colombia. El referido jaguey posee un área de 0,5 ha aproximadamente. El segundo ejemplar (3 de noviembre) fue capturado en un jagüey de similares características, al Sur de Betulia, municipio del mismo nombre que está situado en los $9^{\circ} 15^{\prime} 49.54$ " N y los $75^{\circ} 14^{\prime} 24.41^{\prime \prime}$ O, a una altura s. n. m. de $129 \mathrm{~m}$. Este cuerpo de agua tiene un área aproximada de 31.6 ha. Ambas zonas (Fig. 1 y 2) se clasifican como pertenecientes al bosque seco tropical (HOLDRIDGE, 1967) o como zonobioma tropical alternohigríco (HERNÁNDEZ y SÁNCHEZ, 1992), que estructura grandes extensiones de sabanas antrópicas y que no posee un río o cuenca permanente; presentan en sus orillas abundante vegetación acuática, así como una cobertura densa de Eichornia crassipes (taruya).

BRANDÃO et al. (2003), en un estudio realizado en Brasil con Pseudis pseudis bulbodactyla, registran como área de trabajo una laguna artificial de $180 \mathrm{~m}^{2}$, 
como hábitat de colectas, pero indican que dicho cuerpo de agua era irrigado por un tributario del río Tocantins, Goias; lo que mantiene la afirmación de la ocupación preferencial de zonas pertenecientes a cuencas de ríos, en general para el género Pseudis, aspecto contrario al lugar de hallazgo de los especímenes, ya que se trataba en ambos casos de un jagüey o laguna artificial aislada de cualquier cuerpo de agua corriente, rodeados de praderas ganaderas, como se describió anteriormente.

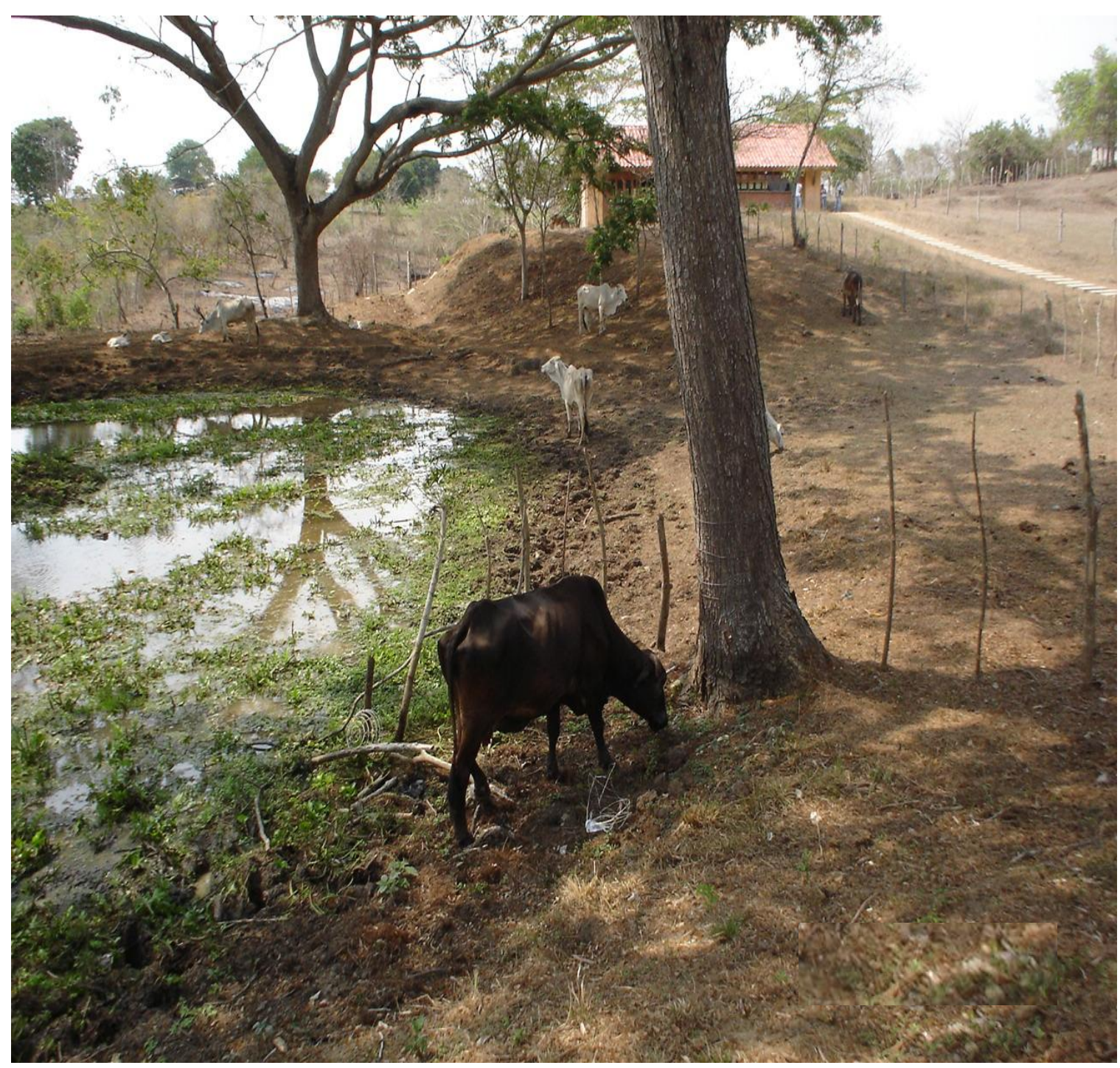

Figura 1. Jagüey en la Granja El Perico, Universidad de Sucre, Colombia, donde fue recolectado el ejemplar de Pseudis paradoxa el 20 de octubre de 2008 


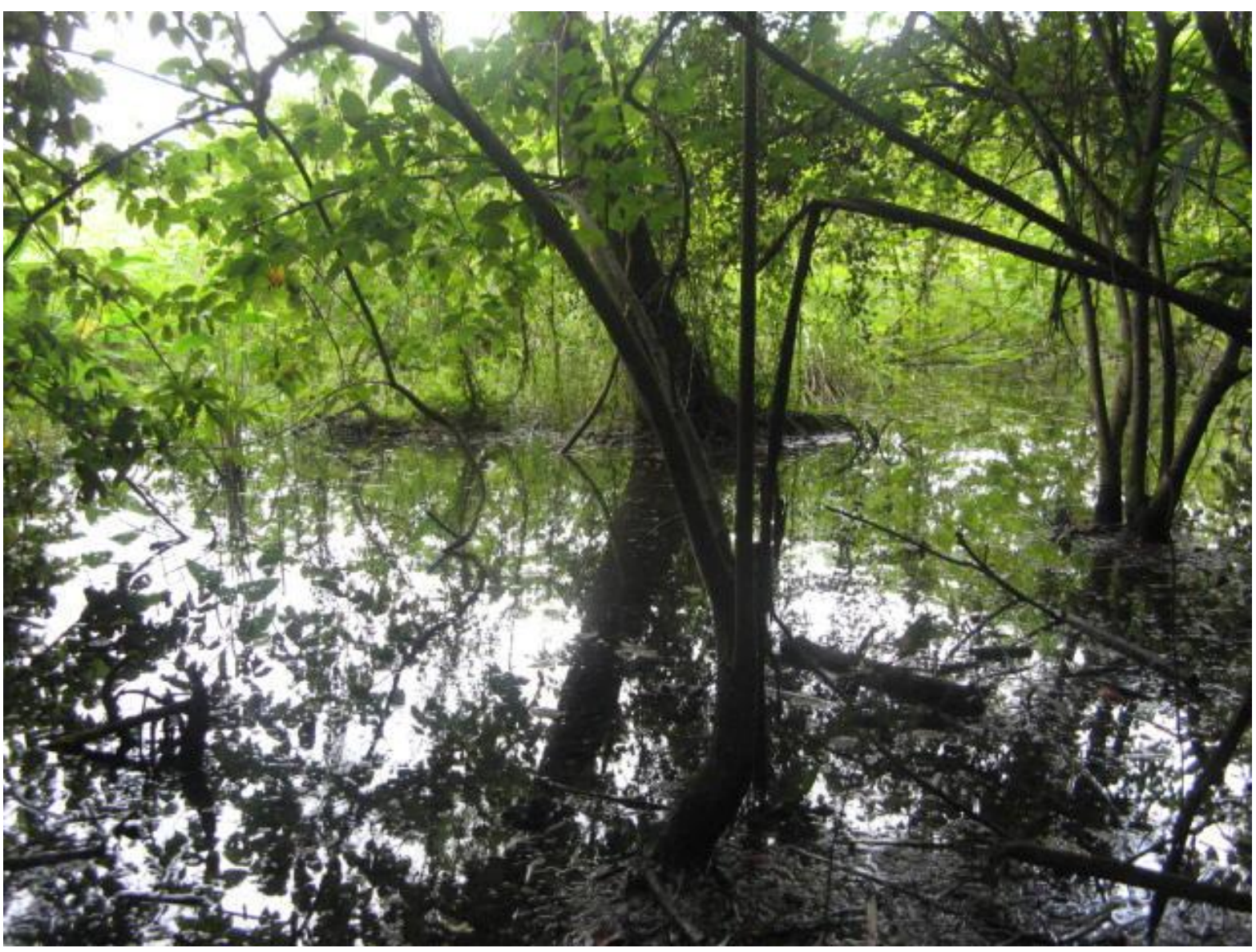

Figura 2. Jagüey al Sur de Betulia, departamento de Sucre, Colombia, donde fue recolectado un ejemplar de Pseudis paradoxa el 4 de noviembre de 2008

Se plantea que el género Pseudis tiene ocurrencia en hábitat acuáticos conformados por los planos de las cuencas de ríos en Venezuela, Colombia, Perú, Bolivia, Paraguay, Uruguay, Argentina y Brasil (DUELLMAN y TRUEB, 1986; MORALES y CHANDLER, 1992); No obstante, la zona de hallazgo concuerda con LYNCH (1979), quien afirma que $P$. paradoxa requiere además de ambientes lénticos para su reproducción y desarrollo, ambientes abiertos en su ciclo de vida, lo cual podría ser una particularidad de esta especie en el Caribe colombiano, donde parece ocupar ambientes secos y cuerpos de agua lóticos, aislados.

En la actualidad el género Pseudis Wagler 1830, comprende dos especies $P$. minuta Gunter 1858 y P. paradoxa Linnaeus 1758; Según GALLARDO (1961); COCHRAN y GOIN (1970), esta última especie se subdivide en siete subespecies: $P$. p. paradoxa, con distribución amazónicamente; $P$. p. fusca en la cuenca del río Jequitinhonha, Minas Gerais, Brasil; $P$. p. bulbodactyla, en las cuencas de los ríos São Francisco y Doce, Minas Gerais, Bahía y Espíritu 
Santo, Brasil; P. p. caribensis, registrada para Trinidad y nordeste de Venezuela; $P$. p. platensis, para las cuencas de los ríos Paraná y Paraguay; $P$. p. occidentalis, registrada en El pailón, Bolivia; y $P$. p. nicefori para el norte de Colombia, que a nivel subespecifico sería la denominación taxonómica que recibirían los especímenes hallados (Fig. 3 y 4 ) y que constituye el primer reporte para el departamento de Sucre.

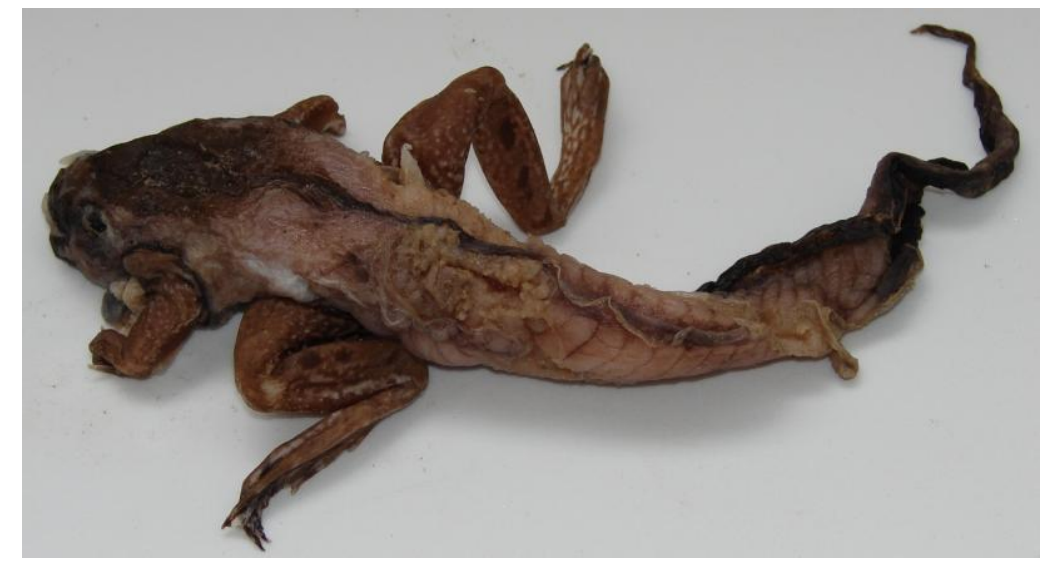

Figura 3. Vista lateral de un ejemplar de P. paradoxa recolectado en la Granja El Perico, Sampués, Sucre. (Foto: Luz Mercedes Botero)

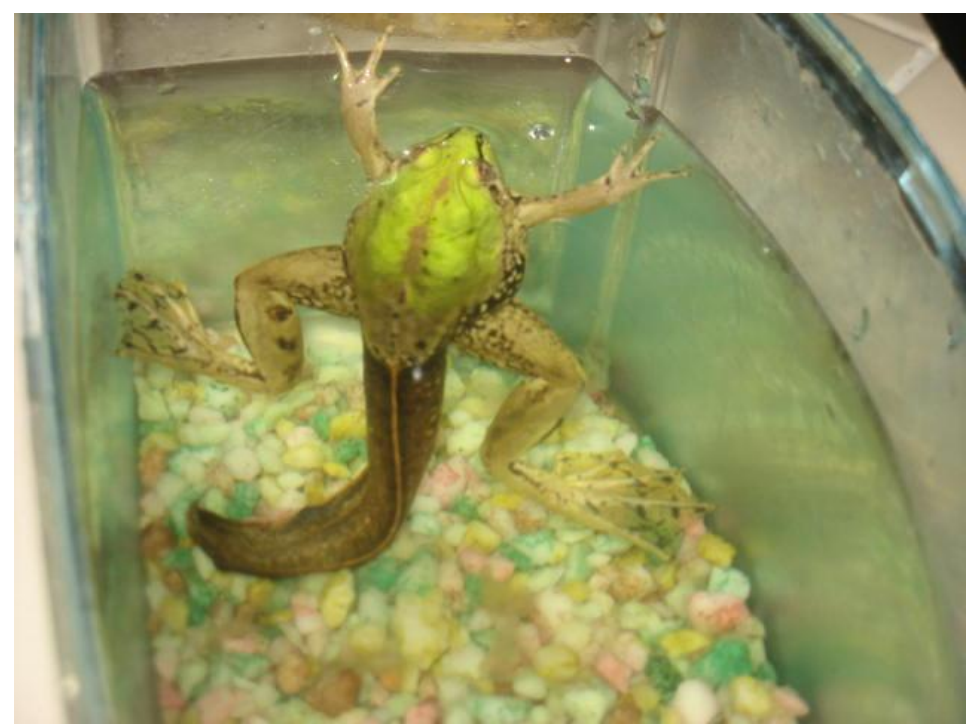

Figura 4. Vista dorsal dentro del agua, en un acuario, de un ejemplar de $P$. paradoxa recolectado al sur de Betulia, Sucre. (Foto: Alcides C. Sampedro Marín)

El ejemplar capturado en el Perico murió muy rápidamente después de su captura. De él se obtuvieron las siguientes medidas $(\mathrm{mm})$ y el peso $(\mathrm{g})$ : longitud total (LT), longitud rostro-anal (LRA), altura del cuerpo (AC), distancia internasal (DIN), distancia narina-ojo (DNO), distancia interorbital (DIO), longitud caudal (LC), altura caudal (AC) (CARAMASCHI y GONÇALVES DA 
CRUZ, 1998) (Tabla 1). Para la verificación del estadio de desarrollo metamórfico se relacionó lo establecido por GOSNER (1960).

Tabla 1. Variables morfómetricas del metamórfico de Pseudis paradoxa recolectado en el Perico.

\begin{tabular}{|l|c|}
\hline \multicolumn{1}{|c|}{ Variable } & Medida \\
\hline Longitud total (LT) (mm) & 203 \\
\hline Longitud rostro-anal (LRA) (mm) & 59 \\
\hline Altura del cuerpo (AC) (mm) & 37 \\
\hline Distancia internasal (DIN) (mm) & 9 \\
\hline Distancia narina-ojo (DNO) (mm) & 10 \\
\hline Distancia interorbital (DIO) (mm) & 15 \\
\hline Altura caudal (AC) (mm) & 31 \\
\hline Longitud caudal (LC) (mm) & 144 \\
\hline Peso (g) & 98 \\
\hline
\end{tabular}

El ejemplar capturado en Betulia estaba en muy buen estado y se mantuvo vivo hasta completar su período metamórfico. Se decidió causarle el menor trauma posible para evitar que muriera o se alterara su período de metamorfosis. De él solo se obtuvo la $L R A=49.4 \mathrm{~mm}$, la $L C=123.2$ y la $L R A$ una vez concluida la metamorfosis fue de $65.4 \mathrm{~mm}$.

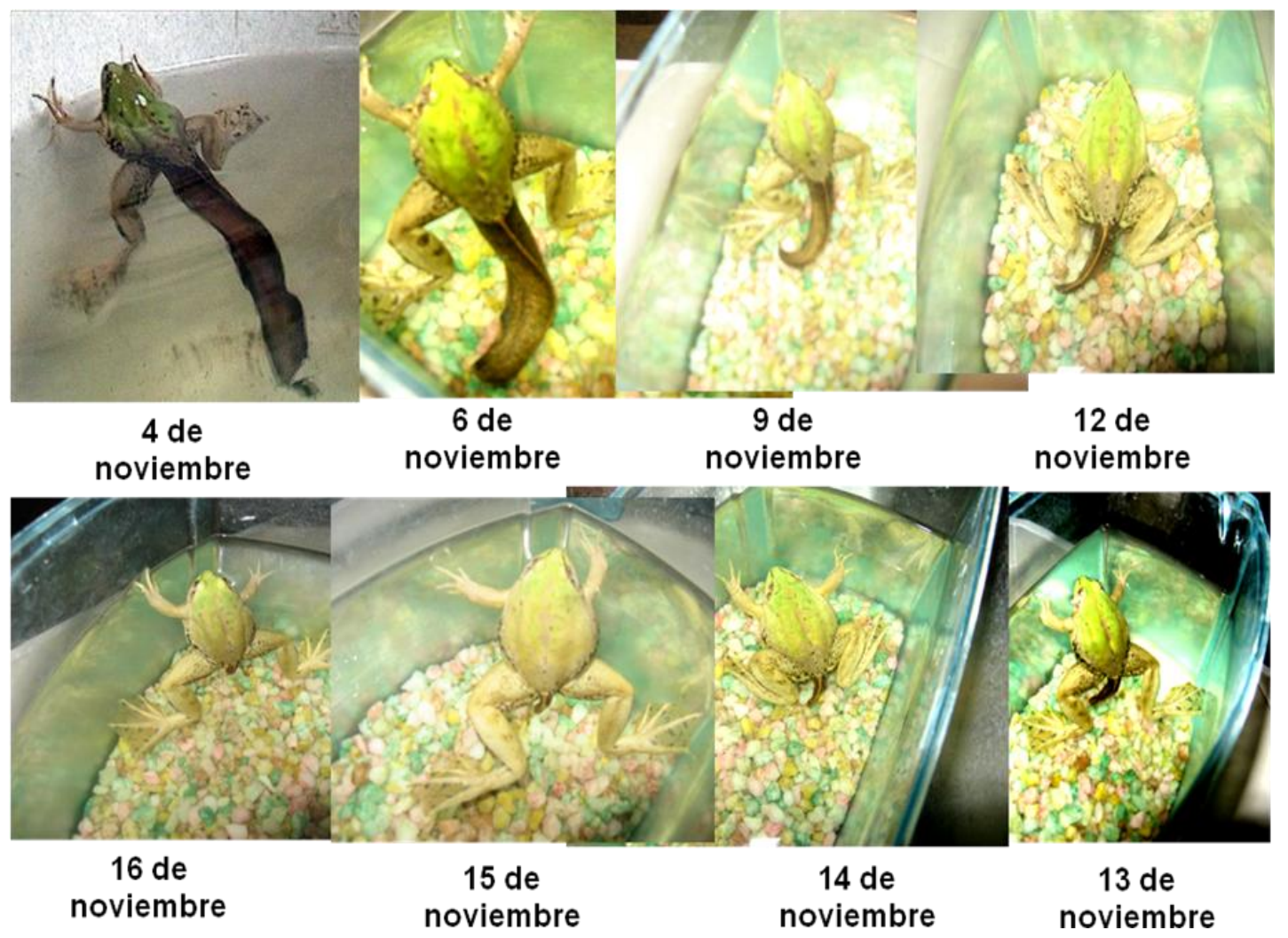

Figura 5. Últimos estadios de la Metamorfosis de Pseudis paradoxa en cautiverio. (Fotos: Alcides C. Sampedro Marín) 
Los renacuajos de esta especie, alcanzan una desproporcionada talla corporal de hasta 220,5 mm (EMERSON, 1988) o hasta 270 mm (BOKERMANN 1967), antes de transformarse en adultos relativamente pequeños (KERH y BASSO, 1990). No existen metamórficos entre las distintas especies de anuros que alcancen una talla comparable (EMERSON, 1988). Según este autor, el gigantismo del metamórfico está relacionado con una extendida exposición a la prolactina; sin embargo, DE SOUZA y KURIBARA (2006), correlacionan el crecimiento de los metamórficos de $P$. paradoxa con múltiples ajustes en la transferencia metabólica del oxigeno en las etapas sucesivas de su metamorfosis.

El ejemplar capturado en Betulia se mantuvo vivo en un acuario de $18 \times 10 \times 14$ $\mathrm{cm}$ y una temperatura atmosférica promedio en el día de $30^{\circ} \mathrm{C}$ y en la noche de $29^{\circ} \mathrm{C}$ y una temperatura del agua promedio durante el día de $29.5^{\circ} \mathrm{C}$ y en la noche de $28.5^{\circ} \mathrm{C}$. Esto permitió determinar que el tiempo de metamorfosis desde el momento de su captura hasta que desapareció totalmente la cola fue de 13 días (Fig. 5). Se requiere obtener ejemplares comenzando su metamorfosis para esclarecer aún más este dato.

De acuerdo con la morfometría se puede establecer que la cola representa el $71 \%$ de la longitud total del espécimen por lo tanto se asume que ambos animales se hallaban en el estadio 38-42, penúltimo de su metamorfosis y en donde alcanza su mayor crecimiento (DE SOUZA y KURIBARA, 2006), aunque el ejemplar capturado en Betulia estaba un poco más avanzado, pero no por muchos días de diferencia. Puede determinarse además que el período reproductivo para esta especie parece comenzar con el segundo período de lluvias en este territorio, que comienza en septiembre y concluye en diciembre. Este individuo fue liberado en el mismo jagüey donde fue recolectado, el día 17 de noviembre de 2008 y tenía un aspecto muy saludable (Fig. 6).

La dieta de $P$. paradoxa consiste de insectos acuáticos y anfibios (CEI, 1980); DURÉ y KEHR (2001), indican que consumen dípteros y anuros. Para forrajeo dependen de la vegetación acuática que coloniza los cuerpos de agua (SAVAGE y CARVALHO, 1953; GALLARDO, 1961; MORALES y CHANDLER, 1992; DIXON et al., 1995; BOSCH et al., 1996, SÁ y LAVILLA, 1997; DE LA RIVA. 1999; KWET, 2000; BRANDÃO y PÉRES Jr. 2001; DURÉ y KEHR, 2001; ARIAS et al., 2002); de manera particular para P. bolbodactyla BRANDÃO et al. (2003) registran ocupación territorial exclusivamente de lagunas con vegetación acuática, lugares propicios para la obtención de su dieta, lo cual coincide en este caso al observar el hábitat de colecta de este metamórfico de $P$. paradoxa. 


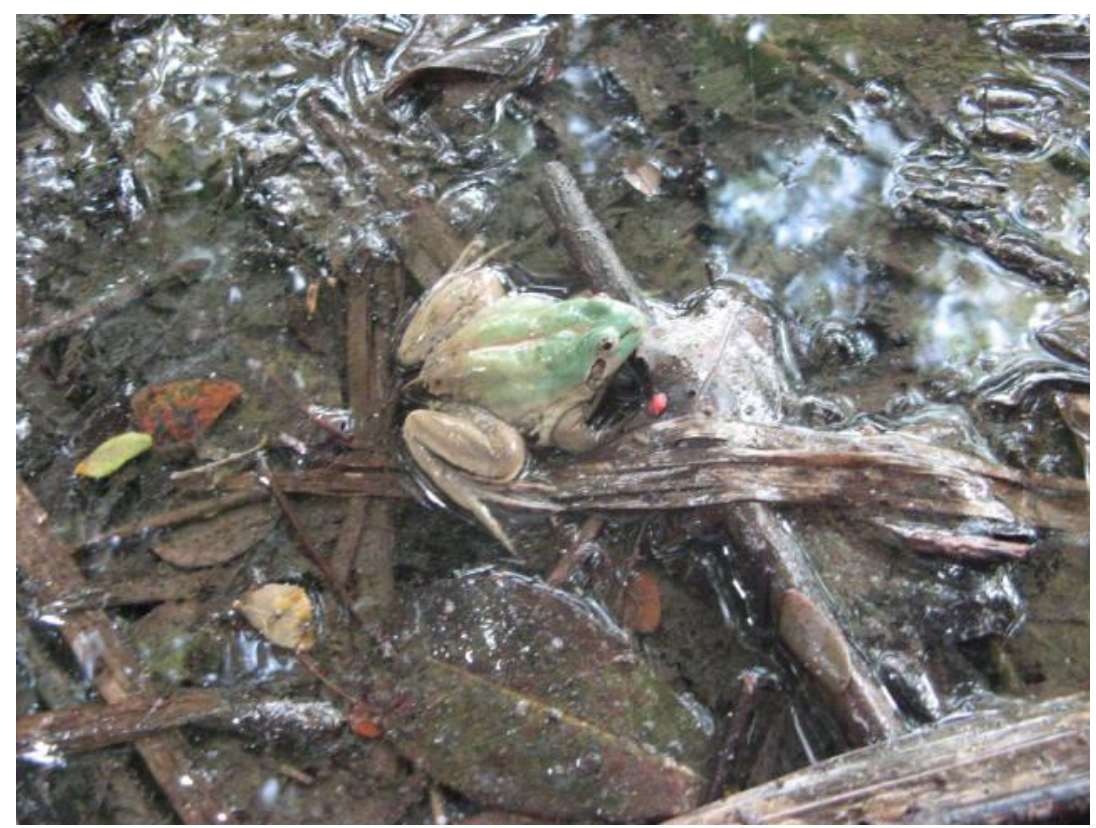

Figura 6. Imagen del ejemplar obtenido en Betulia, en el momento de su liberación en el mismo jagüey donde fuera capturado. (Foto: Alcides C.

Sampedro Marín)

BRANDÃO et al. (2003) señalan como depredadores potenciales para Pseudis. bolbodactyla a: Hoplias malabaricus (moncholo), Casmerodius albus (garza real), Nycticorax nyctycorax (guaco) y Phrynops gibbus (tortuga cabeza de lado); en el caso de las dos localidades señaladas en el presente trabajo, Granja el Perico y Betulia, existen como potenciales depredadores de este anuro, entre otros: Hoplias malabaricus (Moncholo), Ardea alba (garza real), Tigrisoma lineatum (vaco), Caiman crocodilus fuscus (babilla) y Mesoclemmys dahli (carranchina).

Agradecimientos: A Luz Mercedes Botero A. Profesora Facultad de Ciencias Agropecuarias de la Universidad de Sucre, por la ayuda en la toma de las fotografías del ejemplar del Perico y a Iván Sierra M. Director de Fauna Silvestre de CARSUCRE, por facilitar el ejemplar de Betulia.

\section{Referencias}

ARIAS, M. M.; PELTZER, P. M.; LAJMANOVICH, R. C. 2002. Diet of the giant tadpole Pseudis paradoxa platensis (Anura, Pseudidae) from Argentina. Phyllomedusa 1:97-100.

BOKERMANN, W. C. A. 1967. Girinos de anfíbios brasileiros - 3: Sobre um girino gigante de Pseudis paradoxa (Amphibia: Pseudidae). Revista Brasileira de Biologia 27:209-212. 
BOSCH, J.; DE LA RIVA, I.; MÁRQUEZ, R. 1996. The calling behaviour of Lysapsus limellus and Pseudis paradoxa (Amphibia: Anura: Pseudidae). Folia Zoologica 45:49-55.

BRANDÃO, R. A.; GARDA, A.; BRAZ, V.; FONSECA, B. 2003. Observations on the ecology of Pseudis bolbodactyla (Anura, Pseudidae) in central Brazil. Phyllomedusa 2(1):3-8.

BRANDÃO, R. A.; PÉRES JR, A. K. 2001. Levantamento da herpetofauna na área de influência do Aproveitamento Hidroelétrico da UHE Luís Eduardo Magalhães (Palmas, TO). Humanitas 3:35-50.

CARAMASCHI, U.; GONÇALVES DA CRUZ, C. A. 1998. Notas taxonômicas de Pseudis fusca garman e $P$. bolbodactyla a. Lutz, con a descripçao de uma nova espécie correlata (Anura, Pseudidae). Revi. Bras. Zool. 15(4):929-944.

CEI, J. M. 1980. Amphibians of Argentina. Monitore Zoologico Italiano 2:1-609. COCHRAN, D. M.; GOIN, C. J. 1970. Frogs of Colombia. U.S. Nat. Mus. Bull. 288:654-655.

DE LA RIVA, I. 1999. Notes on the paradox frog, Pseudis paradoxa, in Bolivia. British Herpetological Society Bulletin 68:14-19.

DE SOUZA, S. C. R.; KURIBARA, C. M. 2006. Metabolic scaling associated with unusual size changes during larval development of the frog, Pseudis paradoxa. The Journal of Experimental Biology 209:1651-1661.

DIXON, J. R.; MERCOLLI, C.; YANOSKY, A. A. 1995. Some aspects of the ecology of Pseudis paradoxa from northeastern Argentina. Herpetological Review 26:183-185.

DUELLMAN, W. E.; L. TRUEB, L. 1986. Biology of Amphibians. McGraw-Hill. New York.

DURÉ, M. I.; A. I. KHER, A. I. 2001. Differential exploitation of trophic resources by two pseudid frogs from Corrientes, Argentina. Journal of Herpetology 35:340-343.

EMERSON, S. B. 1988. The giant tadpole of Pseudis paradoxa. Biol. J. Linn. Soc. 34:93-104.

GALLARDO, J. M. 1961. On the species of Pseudidae (Amphibia, Anura). Bulletin of the Museum of Comparative Zoology 125:111-134.

GOSNER, K. L. 1960. A simplified table for staging anuran enbryos and larvae with notes on identification. Herpetologica 16:193-189. 
HERNÁNDEZ, C. J.; SÁNCHEZ, P. H. 1992. Biomas terrestre de Colombia. Pág.153-176. En: La biodiversidad biológica de Iberoámerica I. Halffter, G (ed). CYTED, Inst Mex. De Ecología y secretaría de Desarrollo Social. México DF.

HOLDRIDGE, L. 1967. Life zone ecology. Tropical Science Center. San José de Costa Rica.

KERH, A. I.; BASSO, N. G. 1990. Description of the tadpole of Lysapsus limellus (ANURA: PSEUDIDAE) and some considerations on its biology. Copeia 1990(2):573-575.

LYNCH, J.D. 1979. The amphibians of the lowland tropical rainforests. Págs. 189-215 en: Duellman, W.E. (ed.), South American Herpetofauna: Its Origin, Evolution, and Dispersal. The Museum of Natural History. The University of Kansas, Kansas.

MORALES, V. C.; CHANDLER, M. 1992. First record of Pseudis paradoxa (Anura: Pseudidae) in Peru, with notes on its distress call and habitat. Caribbean Journal of Science 28:224-226.

SÁ, R. O.; LAVILLA. E. O. 1997. The tadpole of Pseudis minuta (Anura: Pseudidae), an apparent case of heterochrony. Amphibia-Reptilia 18:229-240.

SAVAGE, J. M.; CARVALHO. A. L. 1953. The family position of neotropical frogs currently referred to the genus Pseudis. Zoologica 38: 193-200. 